AperTO - Archivio Istituzionale Open Access dell'Università di Torino

\title{
Martensen splines and finite-part integrals
}

\section{This is the author's manuscript}

Original Citation:

Availability:

This version is available http://hdl.handle.net/2318/149947

since

Published version:

DOI:10.1007/s11075-014-9921-1

Terms of use:

Open Access

Anyone can freely access the full text of works made available as "Open Access". Works made available under a Creative Commons license can be used according to the terms and conditions of said license. Use of all other works requires consent of the right holder (author or publisher) if not exempted from copyright protection by the applicable law. 


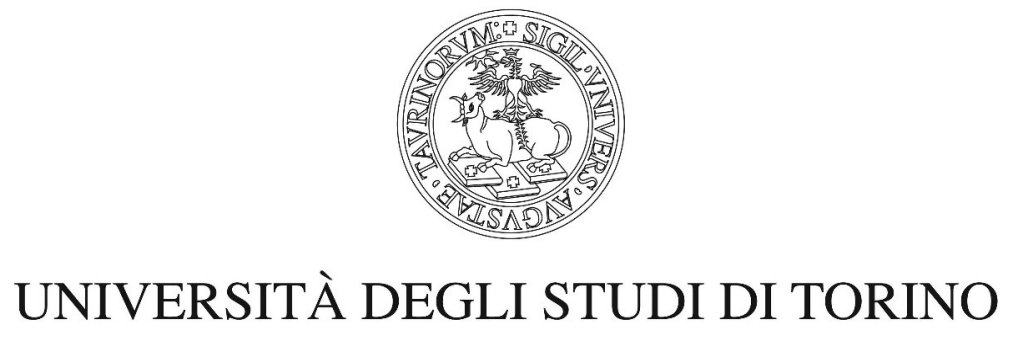

The final publication is available at Springer via

http://dx.doi.org/(DOI 10.1007/s11075-014-9921-1) 


\title{
Martensen splines and finite-part integrals
}

\author{
V. Demichelis and M. Sciarra *
}

\begin{abstract}
We state a uniform convergence theorem for finite-part integrals which are derivatives of weighted Cauchy principal value integrals. We prove that a sequence of Martensen splines, based on locally uniform meshes, satisfies the sufficient conditions required by the theorem. We construct the quadrature rules based on such splines and illustrate their behaviour by presenting some numerical results and comparisons with composite midpoint, Simpson and Newton-Cotes rules.
\end{abstract}

Keywords: Finite-part integral; Uniform convergence; Spline quadrature Subject classification AMS (MOS): 65D07, 65D30, 65D32

\section{Introduction}

We consider the Hadamard finite-part integral

$$
f_{a}^{b} \frac{f(x)}{(x-\lambda)^{p+1}} d x, \lambda \in(a, b), p \in \mathbb{N}
$$

which is well defined for $f \in H_{p, \mu}(B)$, with

$$
H_{p, \mu}(B):=\left\{g \mid g \in C^{p}([a, b]), \omega\left(D^{p} g, \Delta,[a, b]\right) \leq B \Delta^{\mu}, 0<\mu \leq 1, B>0\right\},
$$

where $D^{p}$ denotes the $p$ th derivative operator and

$$
\omega(g, \Delta, J):=\max _{x, x+h \in J, 0 \leq h \leq \Delta}|g(x+h)-g(x)|, g \in C(J) .
$$

For this type of integral the following properties hold [10].

*Department of Mathematics "G. Peano", University of Torino, Via Carlo Alberto 10, I-10123 Torino, Italy (vittoria.demichelis@unito.it, matteosciarra@gmail.com) 
Property 1 When $a<\lambda<b$ and $p \in \mathbb{N}$ we have

$$
f_{a}^{b} \frac{f(x)}{(x-\lambda)^{p+1}} d x=\frac{1}{p} \frac{d}{d \lambda} f_{a}^{b} \frac{f(x)}{(x-\lambda)^{p}} d x=\ldots=\frac{1}{p !} \frac{d^{p}}{d \lambda^{p}} f_{a}^{b} \frac{f(x)}{x-\lambda} d x,
$$

where

$$
\int_{a}^{b} \frac{f(x)}{x-\lambda} d x=\lim _{\epsilon \rightarrow 0}\left\{\int_{a}^{\lambda-\epsilon} \frac{f(x)}{x-\lambda} d x+\int_{\lambda+\epsilon}^{b} \frac{f(x)}{x-\lambda} d x\right\}
$$

is the Cauchy principal value integral.

Property 2 For $c \geq 0$ and $p \geq 1$ we have

$$
f_{\lambda-c h}^{\lambda+h} \frac{f(x)}{(x-\lambda)^{p+1}} d x= \begin{cases}O\left(h^{-p+1}\right), & \text { if } c=1 \text { and } p \text { is even } \\ O\left(h^{-p}\right), & \text { otherwise. }\end{cases}
$$

Hence, the above integral, which is well defined for $h$ fixed, tends to infinity as $h \rightarrow 0$. These integrals are often encountered in several physical and engineering problems [11, 21].

In this paper, we are interested in the numerical evaluation of (1), obtained by replacing $f$ by an approximation $f_{N}$ from a sequence $\left\{f_{N}\right\}$ such that

$$
f_{a}^{b} \frac{f_{N}(x)}{(x-\lambda)^{p+1}} d x
$$

can be evaluated analytically or easily approximated numerically for all $N$. In [13], Rabinowitz proved the following uniform convergence result for weighted finite-part integrals, which is an extension of the uniform convergence result for weighted Cauchy principal value integrals [12].

Theorem 1 Let

$$
I(f ; \lambda ; p):=f_{-1}^{1} \omega_{\alpha \beta}(x) \frac{f(x)}{(x-\lambda)^{p+1}} d x, \lambda \in(-1,1), p \in \mathbb{N},
$$

be the weighted finite-part integral, where

$$
\omega_{\alpha \beta}(x)=(1-x)^{\alpha}(1+x)^{\beta}, \quad \alpha, \beta>-1,
$$

and $f \in H_{p, \mu}(B)$ on $J:=[-1,1]$. Let $\left\{f_{N}\right\}$ be a sequence of approximations of $f$ such that $f_{N} \in C^{p}(J)$ and, setting $e_{N}:=f-f_{N}$,

$$
\begin{gathered}
\left\|D^{j} e_{N}\right\|_{\infty}=o(1) \text { for } N \rightarrow \infty, j=0, \ldots, p, \\
D^{j} e_{N}(-1)=0, \quad 0 \leq j \leq p-\beta, \quad D^{j} e_{N}(1)=0, \quad 0 \leq j \leq p-\alpha, \\
e_{N} \in H_{p, \sigma}\left(B_{1}\right), \quad 0<\sigma \leq \mu, \text { for some } B_{1}>0 \text { and all } N .
\end{gathered}
$$

Then $I\left(f_{N} ; \lambda ; p\right)$ exists and, if $\sigma+\min (\alpha, \beta)>0$, the sequence $\left\{I\left(f_{N} ; \lambda ; p\right)\right\}$ converges uniformly to $I(f ; \lambda ; p)$ for all $\lambda \in(-1,1)$. 
In [4] two examples of sequences $\left\{f_{N}\right\}$ based on locally uniform partitions and satisfying (4)-(6) are provided for any positive integer $p$. These are the modified approximating splines and the modified optimal nodal splines, which are obtained by modifying the approximating splines [8] as well as the optimal nodal splines $[1,2,3]$ in such a way that condition (5) is true for any positive integer $p$. In this paper, we consider sequences of approximating splines for which we can prove (4)-(6) without modifying their definition on $[a, b]$. In particular, we shall consider the Martensen spline operator, introduced in [9] and recently studied in $[15,16]$. For the numerical evaluation of finite-part integral (1) we propose a sequence of Martensen splines of degree $n$ based on locally uniform partitions. We prove that (4)-(6) are true with $1 \leq p<n$. For finite-part integrals (3), with $p \leq n-1$, we construct the quadrature rules based on Martensen splines of degree $n$. The majority of numerical methods for finite-part integrals (1) are based on suitable composite rules, as, for example, midpoint [20], Simpson [21] and Newton-Cotes [19]. Numerical results show that the proposed spline quadratures perform better than composite quadrature rules [19, 20,21]. Indeed, the proposed method requires a less number of integrand function's evaluations compared to the other ones and allows a considerable flexibility in the choice of quadrature nodes.

\section{Martensen splines}

In this section, we give the necessary background material on Martensen splines as presented in $[15,16,17]$ and [5].

Let $T_{R}:=\left\{a=t_{0}<\ldots<t_{R n}=b\right\}$ be a partition of the interval $[a, b]$, we denote by $\Pi_{n}\left(T_{R}\right)$ the linear space of piecewise polynomial functions of degree at most $n$ with breakpoints at $t_{j}$. Let

$$
S_{n+1}\left(T_{R}\right):=\Pi_{n}\left(T_{R}\right) \cap C^{n-1}([a, b])
$$

be the linear space of polynomial splines of degree at most $n$ with simple knots at the points of $T_{R}$. Let

$$
B_{s, n+1}(x):=(-1)^{n+1}\left(t_{s+n+1}-t_{s}\right)\left[t_{s}, \ldots, t_{s+n+1}\right](x-\cdot)_{+}^{n},
$$

be the $(n+1)$ th order normalized B-spline [14], where $\left[t_{s}, \ldots, t_{s+n+1}\right] f$ is the $(n+$ 1)th divided difference and

$$
x_{+}^{r}= \begin{cases}x^{r}, & x \geq 0 \\ 0, & x<0\end{cases}
$$

Let

$$
\varphi_{s, n}(y):=\prod_{v=1}^{n}\left(y-t_{s+v}\right) \in \Pi_{n}
$$


be the dual polynomial for $B_{s, n+1}$. For $i=0, \ldots, n-1$ and $r=0, \ldots, R$ we denote by $F_{i}^{(r n)}$ the Hermite-Martensen splines or HM-splines defined by [5]

$$
F_{i}^{(r n)}(x):=\sum_{s=r n-n}^{r n-1} \frac{(-1)^{i}}{n !} \frac{d^{n-i}}{d y^{n-i}} \varphi_{s, n}\left(t_{r n}\right) B_{s, n+1}(x) \in S_{n+1}\left(T_{R}\right), \quad x \in\left[t_{r n-n}, t_{r n+n}\right],
$$

where $\frac{d^{n-i}}{d y^{n-i}} \varphi_{s, n}\left(t_{r n}\right)$ is the $(n-i)$ th derivative of $\varphi_{s, n}(y)$ evaluated at $y=t_{r n}$.

Assuming that $f \in C^{n-1}([a, b])$, the defining formula for the Martensen spline $M_{R}(f)$ of degree $n$ on $[a, b]$ is given by [17],

$$
M_{R}(f)(x):=\sum_{r=0}^{R} \sum_{i=0}^{n-1} D^{i} f\left(t_{r n}\right) F_{i}^{(r n)}(x) \in S_{n+1}\left(T_{R}\right)
$$

In order to define $F_{i}^{(0)}$ and $F_{i}^{(R n)}$ we need the $2 n$ auxiliary B-spline knots $t_{-n}<$ $\ldots<t_{-1}<t_{0}$ and $t_{R n}<t_{R n+1}<\ldots<t_{R n+n}$.

We introduce the fundamental Hermite splines [16]

$$
\begin{aligned}
& G_{i, n, t_{r n-n}, \ldots, t_{r n}}(x)=\sum_{s=r n-n}^{r n-1} \frac{(-1)^{i}}{n !} \frac{d^{n-i}}{d y^{n-i}} \varphi_{s, n}\left(t_{r n}\right) B_{s, n+1}(x), \quad x \in\left[t_{r n-n}, t_{r n}\right], \\
& H_{i, n, t_{r n}, \ldots, t_{r n+n}}(x)=\sum_{s=r n-n}^{r n-1} \frac{(-1)^{i}}{n !} \frac{d^{n-i}}{d y^{n-i}} \varphi_{s, n}\left(t_{r n}\right) B_{s, n+1}(x), \quad x \in\left[t_{r n}, t_{r n+n}\right] .
\end{aligned}
$$

The HM-splines $F_{i}^{(r n)}$ can be expressed in terms of $G_{i, n, t_{r n-n}, \ldots, t_{r n}}(x)$ and $H_{i, n, t_{r n}, \ldots, t_{r n+n}}(x)$ [16],

$$
F_{i}^{(r n)}(x)=\chi_{\left(-\infty, t_{r n}\right]}(x) G_{i, n, t_{r n-n}, \ldots, t_{r n}}(x)+\chi_{\left[t_{r n}, \infty\right)}(x) H_{i, n, t_{r n}, \ldots, t_{r n+n}}(x) .
$$

Using (9), for $x \in\left[t_{k n}, t_{k n+n}\right] M_{R}(f)(x)$ can be written in the form [15]

$$
M_{R}(f)(x)=\sum_{i=0}^{n-1}\left(D^{i} f\left(t_{k n}\right) H_{i, n, t_{k n}, \ldots, t_{k n+n}}(x)+D^{i} f\left(t_{k n+n}\right) G_{i, n, t_{k n}, \ldots, t_{k n+n}}(x)\right) .
$$

The Martensen spline $M_{R}(f)$ satisfies the interpolation conditions

$$
D^{k} M_{R}(f)\left(t_{r n}\right)=D^{k} f\left(t_{r n}\right), \quad k=0, \ldots, n-1, r=0, \ldots, R,
$$

i.e. $M_{R}(f)$ interpolates certain values of $f$ and its derivatives [16]. So, we can subdivide the knots in primary and secondary ones. In particular,

$$
T_{R, p}:=\left\{t_{r n} \mid r=0, \ldots, R\right\}
$$


is the set of primary knots, whereas

$$
T_{R, s}:=\left\{t_{j} \mid j \neq 0 \bmod n, j=0, \ldots, R n\right\}
$$

is the set of secondary ones.

We denote with

$$
h_{k}:=t_{k n+n}-t_{k n}, \text { with } k=0, \ldots, R-1 \text {, }
$$

and

$$
H_{R}:=\max _{k=0, \ldots, R-1} h_{k} .
$$

The following further properties hold [16]

$$
\begin{gathered}
M_{R}(f)=f \text { for any } f \in S_{n+1}\left(T_{R}\right), \\
M_{R}(f)=f \text { for any } f \in \mathbb{P}^{n} .
\end{gathered}
$$

Let

$$
A_{R}:=\max _{\substack{0 \leq i, j \leq R n-1 \\|i-j|=1}} \frac{t_{i+1}-t_{i}}{t_{j+1}-t_{j}}, \quad A_{R} \geq 1
$$

and

$$
\tilde{A}_{R}:=\max _{\substack{0 \leq i, j \leq R-1 \\|i-j|=1}} \frac{h_{i}}{h_{j}}, \quad \tilde{A}_{R} \geq 1
$$

We say that the sequence of partitions $\left\{T_{R}\right\}_{R \in \mathbb{N}}\left(\left\{T_{R, p}\right\}_{R \in \mathbb{N}}\right)$ is locally uniform if there exists a constant $A \geq 1(\tilde{A} \geq 1)$ such that $A_{R} \leq A\left(\tilde{A}_{R} \leq \tilde{A}\right)$ for all $R$.

Let

$$
e_{R}^{(s)}=D^{s}\left(f-M_{R}(f)\right), \quad 0 \leq s \leq n-1 .
$$

The following uniform convergence result is provided in [5] for Martensen splines and its derivatives.

Theorem 2 Let $f \in C^{n-1}([a, b])$, suppose that $\left\{T_{R, p}\right\}_{R \in \mathbb{N}}$ is such that

$$
H_{R} \rightarrow 0 \text { as } R \rightarrow \infty \text {. }
$$

If $\left\{T_{R, p}\right\}_{R \in \mathbb{N}}$ is locally uniform, then

$$
\left\|e_{R}^{(0)}\right\|_{\infty} \rightarrow 0 \text { as } R \rightarrow \infty .
$$

If $\left\{T_{R}\right\}_{R \in \mathbb{N}}$ is locally uniform, then, for $1 \leq s \leq n-1$,

$$
\left\|e_{R}^{(s)}\right\|_{\infty} \rightarrow 0 \text { as } R \rightarrow \infty .
$$




\section{Martensen splines for numerical evaluation of finite- part integrals}

In order to evaluate (3) numerically with $n$-order singularity at $\lambda$ and $f \in$ $H_{n-1, \mu}(B)$ on $[-1,1]$, we consider the sequence $\left\{M_{R}(f)\right\}$ of Martensen splines of degree $n$, based on a sequence of locally uniform partitions $\left\{T_{R}\right\}$, as approximants for $f$.

The condition (4) of Theorem 1 is true in virtue of Theorem 2. Whereas, condition (5) follows from (10), with $r=0, R$. In order to verify (6), we need the following lemmas. Lemma 1 gives a local estimate for $\left|D^{n} M_{R}(f)(t)\right|$, with $t \in\left[t_{l}, t_{l+1}\right]$. Lemma 2, proved in [5], provides a local estimate for $\left|e_{R}^{(s)}(t)\right|$, with $t \in\left[t_{l}, t_{l+1}\right]$ and $s=0,1, \ldots, n-1$.

Lemma 1 Let $f \in C^{n-1}\left(\left[t_{k n}, t_{k n+n}\right]\right)$, let $t \in\left[t_{l}, t_{l+1}\right] \subset\left[t_{k n}, t_{k n+n}\right]$, then

$$
\left|D^{n} M_{R}(f)(t)\right| \leq K_{l, n} h_{k}^{-1} \omega\left(f^{(n-1)}, h_{k},\left[t_{k n}, t_{k n+n}\right]\right),
$$

where

$$
K_{l, n}:=\frac{2 \Gamma_{n+1, n}}{(n-1) !} \frac{h_{k}^{n}}{\left(t_{l+1}-t_{l}\right)^{n}} \sum_{i=0}^{n-1}(n-i)\left(\begin{array}{c}
n \\
i
\end{array}\right) \tilde{A}_{R}^{i},
$$

with $\tilde{A}_{R}$ defined in (14) and

$$
\Gamma_{n+1, n}:=n !\left(\begin{array}{c}
n \\
{[n / 2]}
\end{array}\right) .
$$

Proof. Let $T_{n-2}(x)$ be the Taylor expansion of $f$ at $t$ and $R_{T}(x)=f(x)-T_{n-2}(x)$. By using (12), we can write

$$
\begin{aligned}
D^{n} M_{R}(f)(x) & =D^{n} M_{R}\left(T_{n-2}+R_{T}\right)(x)=D^{n} M_{R}\left(T_{n-2}\right)(x)+D^{n} M_{R}\left(R_{T}\right)(x) \\
& =D^{n} T_{n-2}(x)+D^{n} M_{R}\left(R_{T}\right)(x)=D^{n} M_{R}\left(R_{T}\right)(x),
\end{aligned}
$$

which yields

$\left|D^{n} M_{R}(f)(t)\right| \leq \sum_{i=0}^{n-1}\left(\left|D^{i} R_{T}\left(t_{k n}\right)\right|\left|D^{n} H_{i, n, t_{k n}, \ldots, t_{k n+n}}(t)\right|+\left|D^{i} R_{T}\left(t_{k n+n}\right)\right|\left|D^{n} G_{i, n, t_{k n}, \ldots, t_{k n+n}}(t)\right|\right)$.

In [5], for $i=0, \ldots, n-1$, the following estimates are proved:

$$
\begin{gathered}
\left|D^{i} R_{T}\left(t_{k n}\right)\right| \leq \frac{h_{k}^{n-i-1}}{(n-i-1) !} \omega\left(f^{(n-1)}, h_{k},\left[t_{k n}, t_{k n+n}\right]\right), \\
\left|D^{n} G_{i, n, t_{k n}, \ldots, t_{k n+n}}(t)\right| \leq \frac{n !}{i !} \frac{\Gamma_{n+1, n}}{(n-1) !} \frac{\tilde{A}_{R}^{i} h_{k}^{i}}{\left(t_{l+1}-t_{l}\right)^{n}} .
\end{gathered}
$$


The estimates (16) and (17) are also true respectively for $\left|D^{i} R_{T}\left(t_{k n+n}\right)\right|$ and $\left|D^{n} H_{i, n, t_{k n}, \ldots, t_{k n+n}}(t)\right|$. Using (16) and (17), we are allowed to conclude that

$$
\begin{aligned}
\left|D^{n} M_{R}(f)(t)\right| & \leq 2 \sum_{i=0}^{n-1} \frac{h_{k}^{n-i-1}}{(n-i-1) !} \omega\left(f^{(n-1)}, h_{k},\left[t_{k n}, t_{k n+n}\right]\right) \frac{n !}{i !} \frac{\Gamma_{n+1, n}}{(n-1) !} \frac{\tilde{A}_{R}^{i} h_{k}^{i}}{\left(t_{l+1}-t_{l}\right)^{n}} \\
& =2 \omega\left(f^{(n-1)}, h_{k},\left[t_{k n}, t_{k n+n}\right]\right) \frac{h_{k}^{n-1}}{\left(t_{l+1}-t_{l}\right)^{n}} \frac{\Gamma_{n+1, n}}{(n-1) !} \sum_{i=0}^{n-1} \frac{\tilde{A}_{R}^{i} n !}{(n-i-1) ! i !} \\
& =K_{l, n} h_{k}^{-1} \omega\left(f^{(n-1)}, h_{k},\left[t_{k n}, t_{k n+n}\right]\right),
\end{aligned}
$$

with $K_{l, n}$ defined in (15).

Lemma 2 Let $t \in\left[t_{l}, t_{l+1}\right] \subset\left[t_{k n}, t_{k n+n}\right]$ and let $f \in C^{n-1}\left(\left[t_{k n}, t_{k n+n}\right]\right)$, then, for $0 \leq s \leq n-1$, we have

$$
\left|e_{R}^{(s)}(t)\right| \leq \begin{cases}K_{0} h_{k}^{n-1} \omega\left(f^{(n-1)}, h_{k},\left[t_{k n}, t_{k n+n}\right]\right), & \text { for } s=0, \\ K_{l, s} h_{k}^{n-1-s} \omega\left(f^{(n-1)}, h_{k},\left[t_{k n}, t_{k n+n}\right]\right), & \text { for } 1 \leq s \leq n-1,\end{cases}
$$

where

$$
\begin{gathered}
K_{0}:=\frac{2}{(n-1) !} \sum_{i=0}^{n-1}(n-i)\left(\begin{array}{c}
n \\
i
\end{array}\right) \tilde{A}_{R}^{i}, \\
K_{l, s}:=\frac{2 \Gamma_{n+1, s}}{(n-1) !} \frac{h_{k}^{s}}{\left(t_{l+1}-t_{l}\right)^{s}} \sum_{i=0}^{n-1}(n-i)\left(\begin{array}{c}
n \\
i
\end{array}\right) \tilde{A}_{R}^{i},
\end{gathered}
$$

with

$$
\Gamma_{n+1, s}:=\frac{n !}{(n-s) !}\left(\begin{array}{c}
s \\
{[s / 2]}
\end{array}\right)
$$

Now, we can prove the following theorem, which provides a bound for the modulus of continuity of $D^{n-1} M_{R}(f)$.

Theorem 3 Let $f \in C^{n-1}(J)$, if $M_{R}(f) \in S_{n+1}\left(T_{R}\right)$ satisfies

$$
\begin{gathered}
\left|e_{R}^{(n-1)}(t)\right| \leq C_{1} \omega\left(f^{(n-1)}, h_{k},\left[t_{k n}, t_{k n+n}\right]\right), \quad t \in\left[t_{l}, t_{l+1}\right], \\
\left|D^{n} M_{R}(f)(t)\right| \leq C_{2} h_{k}^{-1} \omega\left(f^{(n-1)}, h_{k},\left[t_{k n}, t_{k n+n}\right]\right), \quad t \in\left(t_{l}, t_{l+1}\right),
\end{gathered}
$$

with $\left[t_{l}, t_{l+1}\right] \subset\left[t_{k n}, t_{k n+n}\right]$. Then

$$
\omega\left(D^{n-1} M_{R}(f), \Delta, J\right) \leq C_{3} \omega\left(f^{(n-1)}, \Delta, J\right) .
$$


Proof. We have to show that, for $-1 \leq u<v \leq 1$,

$$
\left|D^{n-1} M_{R}(f)(v)-D^{n-1} M_{R}(f)(u)\right| \leq C_{3} \omega\left(f^{(n-1)}, v-u, J\right) .
$$

Assume first that $u, v \in\left[t_{l}, t_{l+1}\right]$. Since $M_{R}(f) \in C^{n-1}\left(\left[t_{l}, t_{l+1}\right]\right)$ and $M_{R}(f) \in$ $C^{n}\left(\left(t_{l}, t_{l+1}\right)\right)$, it follows that

$$
D^{n-1} M_{R}(f)(v)-D^{n-1} M_{R}(f)(u)=(v-u) D^{n} M_{R}(f)(\xi), \quad u<\xi<v .
$$

By condition (19) we get

$$
\begin{aligned}
\left|D^{n-1} M_{R}(f)(v)-D^{n-1} M_{R}(f)(u)\right| & \leq C_{2}|v-u| h_{k}^{-1} \omega\left(f^{(n-1)}, h_{k},\left[t_{k n}, t_{k n+n}\right]\right) \\
& \leq C_{2}|v-u| h_{k}^{-1} \omega\left(f^{(n-1)}, h_{k}, J\right) .
\end{aligned}
$$

Using the following property of the modulus of continuity [18]:

$$
c \omega(g, d, I) \leq 2 d \omega(g, c, I), \text { for } 0<c \leq d,
$$

since $v-u \leq h_{k}$, we have that

$$
\left|D^{n-1} M_{R}(f)(v)-D^{n-1} M_{R}(f)(u)\right| \leq 2 C_{2} \omega\left(f^{(n-1)},|v-u|, J\right) .
$$

If $t_{l} \leq u \leq t_{l+1}<v \leq t_{l+2}$, using (21) we obtain

$$
\begin{aligned}
\left|D^{n-1} M_{R}(f)(v)-D^{n-1} M_{R}(f)(u)\right| \leq & \left|D^{n-1} M_{R}(f)(v)-D^{n-1} M_{R}(f)\left(t_{l+1}\right)\right| \\
& +\left|D^{n-1} M_{R}(f)\left(t_{l+1}\right)-D^{n-1} M_{R}(f)(u)\right| \\
\leq & 4 C_{2}|v-u| \omega\left(f^{(n-1)},|v-u|, J\right) .
\end{aligned}
$$

Finally, we consider $t_{k_{1} n} \leq t_{i} \leq u \leq t_{i+1} \leq t_{k_{1} n+n}$ and $t_{k_{2} n} \leq t_{j} \leq v \leq t_{j+1} \leq t_{k_{2} n+n}$, with $j>i+1$ and $k_{1} \leq k_{2}$. It can be easily proved that

$$
t_{k n+n}-t_{k n} \leq\left(t_{l+1}-t_{l}\right)\left(\sum_{j=k n}^{l} A_{R}^{l-j}+\sum_{j=l+1}^{k n+n-1} A_{R}^{j-l}\right)
$$

where $A_{R}$ is given in (13). From (22) and the fact that

$$
\left(t_{i+2}-t_{i+1}\right),\left(t_{j}-t_{j-1}\right) \leq v-u
$$

(since $\left[t_{i+1}, t_{i+2}\right],\left[t_{j-1}, t_{j}\right] \subseteq[u, v]$ ), we get

$$
t_{k_{1} n+n}-t_{k_{1} n} \leq(v-u)\left(\sum_{m=k_{1} n}^{i+1} A_{R}^{i+1-m}+\sum_{m=i+2}^{k_{1} n+n-1} A_{R}^{m-i-1}\right),
$$




$$
t_{k_{2} n+n}-t_{k_{2} n} \leq(v-u)\left(\sum_{m=k_{2} n}^{j-1} A_{R}^{j-1-m}+\sum_{m=j}^{k_{2} n+n-1} A_{R}^{m-j+1}\right) .
$$

We can notice that

$$
\begin{aligned}
\left|D^{n-1} M_{R}(f)(v)-D^{n-1} M_{R}(f)(u)\right| \leq & \left|f^{(n-1)}(v)-D^{n-1} M_{R}(f)(v)\right| \\
& +\left|f^{(n-1)}(u)-D^{n-1} M_{R}(f)(u)\right| \\
& +\left|f^{(n-1)}(v)-f^{(n-1)}(u)\right| .
\end{aligned}
$$

Obviously,

$$
\left|f^{(n-1)}(v)-f^{(n-1)}(u)\right| \leq \omega\left(f^{(n-1)}, v-u, J\right) .
$$

From (18), (23), (24) and the fact that

$$
\omega\left(g, \delta_{1}+\delta_{2}, I\right) \leq \omega\left(g, \delta_{1}, I\right)+\omega\left(g, \delta_{2}, I\right),
$$

we get

$$
\begin{aligned}
\left|f^{(n-1)}(u)-D^{n-1} M_{R}(f)(u)\right| & \leq C_{1} \omega\left(f^{(n-1)}, h_{k_{1}},\left[t_{k_{1} n}, t_{k_{1} n+n}\right]\right) \\
& \leq C_{1}\left(\sum_{m=k_{1} n}^{i+1} A_{R}^{i-m+1}+\sum_{m=i+2}^{k_{1} n+n-1} A_{R}^{m-i-1}\right) \omega\left(f^{(n-1)}, v-u, J\right) \\
& =C_{4} \omega\left(f^{(n-1)}, v-u, J\right),
\end{aligned}
$$

with

$$
C_{4}=C_{1}\left(\sum_{m=k_{1} n}^{i+1} A_{R}^{i-m+1}+\sum_{m=i+2}^{k_{1} n+n-1} A_{R}^{m-i-1}\right)
$$

and, similarly,

$$
\begin{aligned}
\left|f^{(n-1)}(v)-D^{n-1} M_{R}(f)(v)\right| & \leq C_{1} \omega\left(f^{(n-1)}, h_{k_{2}},\left[t_{k_{2} n}, t_{k_{2} n+n}\right]\right) \\
& \leq C_{1}\left(\sum_{m=k_{2} n}^{j-1} A_{R}^{j-m-1}+\sum_{m=j}^{k_{2} n+n-1} A_{R}^{m-j+1}\right) \omega\left(f^{(n-1)}, v-u, J\right) \\
& =C_{5} \omega\left(f^{(n-1)}, v-u, J\right),
\end{aligned}
$$

with

$$
C_{5}=C_{1}\left(\sum_{m=k_{2} n}^{j-1} A_{R}^{j-m-1}+\sum_{m=j}^{k_{2} n+n-1} A_{R}^{m-j+1}\right) .
$$

Then, we get

$$
\left|D^{n-1} M_{R}(f)(v)-D^{n-1} M_{R}(f)(u)\right| \leq\left(1+C_{4}+C_{5}\right) \omega\left(f^{(n-1)}, v-u, J\right) .
$$


This proves our theorem with $C_{3}=\max \left(4 C_{2}, 1+C_{4}+C_{5}\right)$.

Hence, by using (20), we have

$$
\begin{aligned}
\omega\left(e_{R}^{(n-1)}, \Delta, J\right) & \leq \omega\left(f^{(n-1)}, \Delta, J\right)+\omega\left(D^{n-1} M_{R}(f), \Delta, J\right) \\
& \leq\left(1+C_{3}\right) \omega\left(f^{(n-1)}, \Delta, J\right) \leq\left(1+C_{3}\right) B \Delta^{\mu},
\end{aligned}
$$

then $e_{R}^{(0)} \in H_{n-1, \mu}\left(\left(1+C_{3}\right) B\right)$ and condition (6) is satisfied.

By Theorem 2, (10) and (25), the sequence $\left\{M_{R}(f)\right\}$, constructed on a locally uniform sequence of partitions $\left\{T_{R}\right\}$, satisfies conditions (4), (5) and (6), then it is a viable candidate for $\left\{f_{N}\right\}$ in Theorem 1 .

\section{Quadrature rules based on $M_{R}$}

\subsection{General case}

We are interested in evaluating numerically $I(f ; \lambda ; n-1)$, defined in (3), by replacing $f \in C^{n-1}([-1,1])$ with its spline approximation $M_{R}(f)$ of degree $n$, defined in (8). We approximate $I(f ; \lambda ; n-1)$ by the quadrature sum

$$
I(f ; \lambda ; n-1) \cong \sum_{r=0}^{R} \sum_{i=0}^{n-1} D^{i} f\left(t_{r n}\right) w_{r, i}(\lambda),
$$

where

$$
w_{r, i}(\lambda)=\frac{1}{(n-1) !} \frac{d^{n-1}}{d \lambda^{n-1}} f_{-1}^{1} \frac{\omega_{\alpha \beta}(x)}{x-\lambda} F_{i}^{(r n)}(x) d x, \quad r=0, \ldots, R, i=0, \ldots, n-1 .
$$

Using (7), we can write

$$
w_{r, i}(\lambda)=\sum_{s=r n-n}^{r n-1} \frac{(-1)^{i}}{n !} \frac{d^{n-i}}{d y^{n-i}} \varphi_{s, n}\left(t_{r n}\right) \frac{1}{(n-1) !} \frac{d^{n-1}}{d \lambda^{n-1}} f_{-1}^{1} \omega_{\alpha \beta}(x) \frac{B_{s, n+1}(x)}{x-\lambda} d x .
$$

Hence, in order to evaluate $w_{r, i}$, we have to compute

$$
I\left(B_{s, n+1} ; \lambda ; n-1\right)= \begin{cases}\frac{1}{(n-1) !} \frac{d^{n-1}}{d \lambda^{n-1}} \int_{-1}^{t_{s+n+1}} \omega_{\alpha \beta}(x) \frac{B_{s, n+1}(x)}{x-\lambda} d x, \quad s=-n, \ldots,-1, \\ \frac{1}{(n-1) !} \frac{d^{n-1}}{d \lambda^{n-1}} \int_{t_{s}}^{t_{s+n+1}} \omega_{\alpha \beta}(x) \frac{B_{s, n+1}(x)}{x-\lambda} d x, \quad s=0, \ldots, R n-n-1, \\ \frac{1}{(n-1) !} \frac{d^{n-1}}{d \lambda^{n-1}} \int_{t_{s}}^{1} \omega_{\alpha \beta}(x) \frac{B_{s, n+1}(x)}{x-\lambda} d x, & s=R n-n, \ldots, R n-1 .\end{cases}
$$


In order to do this, we can consider all $n$-degree polynomials $p_{j}^{s}(x), j=$ $s, \ldots, s+n$, such that

$$
p_{j}^{s}(x)=B_{s, n+1}(x), \quad x \in\left[t_{j}, t_{j+1}\right]
$$

Setting

$$
p_{j}^{s}(x)=\sum_{k=1}^{n+1} \bar{a}_{k} x^{n+1-k}
$$

we evaluate $p_{j}^{s}(x) /(x-\lambda)$ by using

$$
p_{j}^{s}(x)=(x-\lambda) \sum_{k=1}^{n}\left(\sum_{v=1}^{k} \bar{a}_{v} \lambda^{k-v}\right) x^{n-k}+\sum_{k=1}^{n+1} \bar{a}_{k} \lambda^{n+1-k} .
$$

Using (26), the evaluation of $I\left(B_{s, n+1} ; \lambda ; n-1\right)$ is reduced to the computation of the following integrals:

$$
\begin{aligned}
\frac{d^{n-1}}{d \lambda^{n-1}} \int_{t_{j}}^{t_{j+1}} \omega_{\alpha \beta}(x) \frac{p_{j}^{s}(x)}{x-\lambda} d x= & \frac{d^{n-1}}{d \lambda^{n-1}}\left[\sum_{k=1}^{n}\left(\sum_{v=1}^{k} \bar{a}_{v} \lambda^{k-v}\right) \int_{t_{j}}^{t_{j+1}} \omega_{\alpha \beta}(x) x^{n-k} d x\right. \\
& \left.+\sum_{k=1}^{n+1} \bar{a}_{k} \lambda^{n+1-k} \int_{t_{j}}^{t_{j+1}} \omega_{\alpha \beta}(x) \frac{1}{x-\lambda} d x\right] .
\end{aligned}
$$

In particular, we consider $\alpha=\beta=-0.5,0,0.5$, for which the integrals in (27) can be evaluated exactly, otherwise we have to use a numerical method [6].

\subsection{Singularity of order 2}

We evaluate numerically $I(f ; \lambda ; 1)$ with a 2 -order singularity at $x=\lambda$. Replacing $f$ by

$$
M_{R}(f)(x)=\sum_{r=0}^{R} \sum_{i=0}^{2} D^{i} f\left(t_{3 r}\right) F_{i}^{(3 r)}(x) \in S_{4}\left(T_{R}\right)
$$

we get

$$
I(f ; \lambda ; 1) \cong \sum_{r=0}^{R} \sum_{i=0}^{2} D^{i} f\left(t_{3 r}\right) w_{r, i}(\lambda)
$$

with

$$
w_{r, i}(\lambda)=\frac{d}{d \lambda} \int_{-1}^{1} \frac{\omega_{\alpha \beta}(x)}{x-\lambda} F_{i}^{(3 r)}(x) d x, \quad r=0, \ldots, R, i=0,1,2 .
$$

Using (7) we have

$$
w_{r, i}(\lambda)=\sum_{s=3 r-3}^{3 r-1} \frac{(-1)^{i}}{3 !} \frac{d^{3-i}}{d y^{3-i}} \varphi_{s, n}\left(t_{3 r}\right) \frac{d}{d \lambda} f_{-1}^{1} \omega_{\alpha \beta}(x) \frac{B_{s, 4}(x)}{x-\lambda} d x,
$$


then, to compute $w_{r, i}$, we have to evaluate the following integrals:

$$
I\left(B_{s, 4} ; \lambda ; 1\right)= \begin{cases}\frac{d}{d \lambda} \int_{-1}^{t_{s+4}} \omega_{\alpha \beta}(x) \frac{B_{s, 4}(x)}{x-\lambda} d x, & s=-3,-2,-1, \\ \frac{d}{d \lambda} \int_{t_{s}}^{t_{s+4}} \omega_{\alpha \beta}(x) \frac{B_{s, 4}(x)}{x-\lambda} d x, & s=0, \ldots, 3 R-4, \\ \frac{d}{d \lambda} f_{t_{s}}^{1} \omega_{\alpha \beta}(x) \frac{B_{s, 4}(x)}{x-\lambda} d x, & s=3 R-3,3 R-2,3 R-1 .\end{cases}
$$

Let

$$
p_{j}^{s}(x)=\bar{a}_{1} x^{3}+\bar{a}_{2} x^{2}+\bar{a}_{3} x+\bar{a}_{4}=B_{s, 4}(x), \quad x \in\left[t_{j}, t_{j+1}\right],
$$

with $j=s, s+1, s+2, s+3$, be the polynomial representation of $B_{s, 4}(x)$ in $\left[t_{j}, t_{j+1}\right]$. In order to evaluate the quantities $p_{j}^{s}(x) /(x-\lambda)$, we write $p_{j}^{s}(x)$ in the form

$p_{j}^{s}(x)=\left[\bar{a}_{1} x^{2}+\left(\bar{a}_{2}+\bar{a}_{1} \lambda\right) x+\left(\bar{a}_{3}+\bar{a}_{2} \lambda+\bar{a}_{1} \lambda^{2}\right)\right](x-\lambda)+\bar{a}_{4}+\bar{a}_{3} \lambda+\bar{a}_{2} \lambda^{2}+\bar{a}_{1} \lambda^{3}$.

Then, the evaluation of $I\left(B_{s, 4} ; \lambda ; 1\right)$ is reduced to the computation of the following integrals, for $j=s, s+1, s+2, s+3$,

$$
\begin{aligned}
\frac{d}{d \lambda} f_{t_{j}}^{t_{j+1}} \omega_{\alpha \beta}(x) \frac{p_{j}^{s}(x)}{x-\lambda} d x= & \bar{b}_{1} \int_{t_{j}}^{t_{j+1}} \omega_{\alpha \beta}(x) x d x+\bar{b}_{2} \int_{t_{j}}^{t_{j+1}} \omega_{\alpha \beta}(x) d x \\
& +\bar{b}_{3} \int_{t_{j}}^{t_{j+1}} \omega_{\alpha \beta}(x) \frac{1}{x-\lambda} d x+\bar{b}_{4} \frac{d}{d \lambda} f_{t_{j}}^{t_{j+1}} \omega_{\alpha \beta}(x) \frac{1}{x-\lambda} d x,
\end{aligned}
$$

where

$$
\begin{aligned}
& \bar{b}_{1}=\bar{a}_{1}, \\
& \bar{b}_{2}=\bar{a}_{2}+2 \bar{a}_{1} \lambda, \\
& \bar{b}_{3}=\bar{a}_{3}+2 \bar{a}_{2} \lambda+3 \bar{a}_{1} \lambda^{2}, \\
& \bar{b}_{4}=\bar{a}_{4}+\bar{a}_{3} \lambda+\bar{a}_{2} \lambda^{2}+\bar{a}_{1} \lambda^{3} .
\end{aligned}
$$

\subsection{Singularity of order 3}

Similarly to the previous case, in order to evaluate numerically $I(f ; \lambda ; 2)$, with a 3-order singularity at $x=\lambda$, we replace $f$ by (28). Hence, we get

$$
I(f ; \lambda ; 2) \cong \sum_{r=0}^{R} \sum_{i=0}^{2} D^{i} f\left(t_{3 r}\right) w_{r, i}(\lambda)
$$


with

$$
w_{r, i}(\lambda)=\frac{1}{2} \frac{d^{2}}{d \lambda^{2}} f_{-1}^{1} \frac{\omega_{\alpha \beta}(x)}{x-\lambda} F_{i}^{(3 r)}(x) d x, \quad r=0, \ldots, R, i=0,1,2
$$

Using (7), $w_{r, i}(x)$ can be written in the form

$$
w_{r, i}(\lambda)=\sum_{s=3 r-3}^{3 r-1} \frac{(-1)^{i}}{3 !} \frac{d^{3-i}}{d y^{3-i}} \varphi_{s, n}\left(t_{3 r}\right) \frac{1}{2} \frac{d^{2}}{d \lambda^{2}} \int_{-1}^{1} \omega_{\alpha \beta}(x) \frac{B_{s, 4}(x)}{x-\lambda} d x,
$$

then, in order to evaluate $w_{r, i}$, we have to compute

$$
I\left(B_{s, 4} ; \lambda ; 2\right)= \begin{cases}\frac{1}{2} \frac{d^{2}}{d \lambda^{2}} \int_{-1}^{t_{s+4}} \omega_{\alpha \beta}(x) \frac{B_{s, 4}(x)}{x-\lambda} d x, & s=-3,-2,-1, \\ \frac{1}{2} \frac{d^{2}}{d \lambda^{2}} \int_{t_{s}}^{t_{s+4}} \omega_{\alpha \beta}(x) \frac{B_{s, 4}(x)}{x-\lambda} d x, & s=0, \ldots, 3 R-4, \\ \frac{1}{2} \frac{d^{2}}{d \lambda^{2}} f_{t_{s}}^{1} \omega_{\alpha \beta}(x) \frac{B_{s, 4}(x)}{x-\lambda} d x, & s=3 R-3,3 R-2,3 R-1 .\end{cases}
$$

Let $p_{j}^{s}(x)$ be the polynomial representation of $B_{s, 4}(x)$ in $\left[t_{j}, t_{j+1}\right]$, as shown in Section 4.2 the evaluation of $B_{s, 4}(x)$ is reduced to the computation of the following integrals, for $j=s, s+1, s+2, s+3$,

$$
\begin{aligned}
\frac{1}{2} \frac{d^{2}}{d \lambda^{2}} \int_{t_{j}}^{t_{j+1}} \omega_{\alpha \beta}(x) \frac{p_{j}^{s}(x)}{x-\lambda} d x= & \bar{c}_{1} \int_{t_{j}}^{t_{j+1}} \omega_{\alpha \beta}(x) d x+\bar{c}_{2} f_{t_{j}}^{t_{j+1}} \omega_{\alpha \beta}(x) \frac{1}{x-\lambda} d x \\
& +\bar{c}_{3} \frac{d}{d \lambda} f_{t_{j}}^{t_{j+1}} \omega_{\alpha \beta}(x) \frac{1}{x-\lambda} d x \\
& +\bar{c}_{4} \frac{d^{2}}{d \lambda^{2}} \int_{t_{j}}^{t_{j+1}} \omega_{\alpha \beta}(x) \frac{1}{x-\lambda} d x
\end{aligned}
$$

where

$$
\begin{aligned}
& \bar{c}_{1}=\bar{a}_{1}, \\
& \bar{c}_{2}=\bar{a}_{2}+3 \bar{a}_{1} \lambda, \\
& \bar{c}_{3}=\bar{a}_{3}+2 \bar{a}_{2} \lambda+3 \bar{a}_{1} \lambda^{2}, \\
& \bar{c}_{4}=\frac{1}{2}\left(\bar{a}_{4}+\bar{a}_{3} \lambda+\bar{a}_{2} \lambda^{2}+\bar{a}_{1} \lambda^{3}\right) .
\end{aligned}
$$




\section{Comparisons and numerical examples}

In this section we compare our quadratures (29) and (30) with composite midpoint [20], Simpson [21] and Newton-Cotes [19].

We denote by $E_{R}$ the errors obtained with our quadrature rules,

$$
E_{R}=I\left(e_{R}^{(0)} ; \lambda ; p\right)=I(f ; \lambda ; p)-I\left(M_{R}(f) ; \lambda ; p\right), \quad p=1,2 .
$$

For both values of $p$ we approximate $f$ by the cubic Martensen spline $M_{R}(f)$.

\subsection{Comparison with midpoint}

We evaluate numerically the finite-part integral (3), with $\alpha=\beta=0, p=1$, $\lambda=0$ and

$$
f(x)=x^{2}+x+\left[2+\frac{x}{|x|}\right]|x|^{\gamma+1 / 2}, \quad \gamma=2,3,
$$

by using the quadrature sum (29) and the composite midpoint rule [20]. Obviously, $f(x) \in C^{\gamma+1 / 2}([-1,1])$. The exact value of the finite-part integral is [20]

$$
I(f ; 0 ; 1)=2+\frac{4}{1 / 2+\gamma-1}, \quad \gamma=2,3 .
$$

We adopt a uniform mesh for both methods. Let $E_{R}^{M}$ be the quadrature error obtained by the composite midpoint rule, where $R$ is the number of integration's subintervals.

In Tables 1 and 2 we compare the numerical results presented in [20] with the results obtained by our method, respectively in the case $\gamma=2$ and $\gamma=3$.

With $\gamma=2$ our method performs better than the one presented in [20], whereas with $\gamma=3$ our method is more accurate up to $R=1023$, while for higher values of $R$, the errors grow.

In Fig. 1 the absolute errors, reported in Tables 1 and 2, are represented for the different values of $R$, so we can compare graphically the behaviour of quadrature errors. In both cases $\gamma=2$ and $\gamma=3$ we can see that our method get the same accuracy of midpoint for considerably less values of $R$.

\subsection{Comparison with Simpson}

We evaluate numerically the finite-part integral (3) with $\alpha=\beta=0, p=2$ and

$$
f(x)=x^{4},
$$


Table 1: Errors in the case $\gamma=2$.

\begin{tabular}{lll}
\hline $\mathrm{R}$ & $\left|E_{R}^{M}\right|$ & $\left|E_{R}\right|$ \\
\hline 7 & - & $8.19 \mathrm{e}-02$ \\
15 & - & $2.61 \mathrm{e}-02$ \\
31 & - & $8.79 \mathrm{e}-03$ \\
63 & - & $3.03 \mathrm{e}-03$ \\
127 & - & $1.06 \mathrm{e}-03$ \\
255 & $1.33 \mathrm{e}-03$ & $3.73 \mathrm{e}-04$ \\
511 & $4.86 \mathrm{e}-04$ & $1.31 \mathrm{e}-04$ \\
1023 & $1.76 \mathrm{e}-04$ & $4.65 \mathrm{e}-05$ \\
2047 & $6.31 \mathrm{e}-05$ & $1.46 \mathrm{e}-05$ \\
4095 & $2.26 \mathrm{e}-05$ & $8.00 \mathrm{e}-06$ \\
\hline
\end{tabular}

Table 2: Errors in the case $\gamma=3$.

\begin{tabular}{lll}
\hline $\mathrm{R}$ & $\left|E_{R}^{M}\right|$ & $\left|E_{R}\right|$ \\
\hline 7 & - & $6.31 \mathrm{e}-03$ \\
15 & - & $9.40 \mathrm{e}-04$ \\
31 & - & $1.53 \mathrm{e}-04$ \\
63 & - & $2.60 \mathrm{e}-05$ \\
127 & - & $4.51 \mathrm{e}-06$ \\
255 & $7.07 \mathrm{e}-05$ & $7.89 \mathrm{e}-07$ \\
511 & $1.81 \mathrm{e}-05$ & $1.23 \mathrm{e}-07$ \\
1023 & $4.58 \mathrm{e}-06$ & $1.37 \mathrm{e}-07$ \\
2047 & $1.16 \mathrm{e}-06$ & $1.65 \mathrm{e}-06$ \\
4095 & $2.92 \mathrm{e}-07$ & $2.02 \mathrm{e}-06$ \\
\hline
\end{tabular}



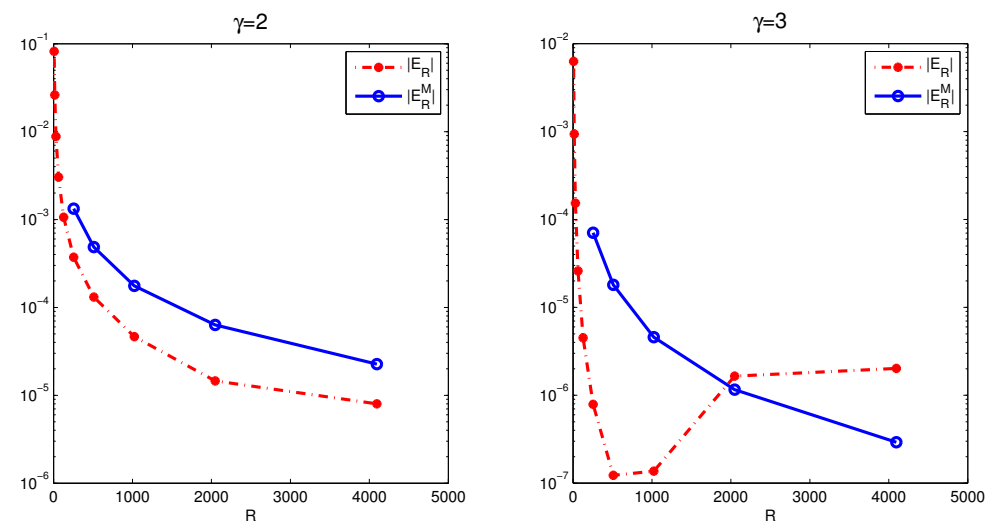

Figure 1: Graphical representation of absolute errors. Axis $y$ is in logarithmic scale.

by using the quadrature sum (30) and the composite Simpson's rule [21].

For both methods we adopt a uniform mesh. We consider two different singularities of order $3, \lambda=t_{[R / 4]}+h / 2+\tau h$ and $\lambda=t_{0}+h / 2+\tau h$, with $\tau=0,1 / 4$, where $h$ is the width of every subinterval. The exact value of the finite-part integral is [21]

$$
I(f ; \lambda ; 2)=6 \lambda-\frac{8 \lambda^{3}-6 \lambda^{5}}{\left(1-\lambda^{2}\right)^{2}}+6 \lambda^{2} \ln \left|\frac{1-\lambda}{1+\lambda}\right| .
$$

In order to construct the composite Simpson's rule, we have to introduce a quadrature node at each subinterval then, to compare our method with Simpson, we have to assume $R=2 n$, where $n$ is the number of subintervals used for the construction of the composite rule.

Let $E_{R}^{S}$ be the quadrature error obtained using the composite Simpson's rule, with $R+1$ quadrature nodes.

In Table 3 we compare the results presented in [21] with the results obtained by our method, in the case $\lambda=t_{[R / 4]}+h / 2+\tau h$, with $\tau=0,1 / 4$, whereas in Table 4 we compare the results obtained by the two methods in the case $\lambda=t_{0}+h / 2+\tau h$, with $\tau=0,1 / 4$.

We represented in Fig. 2 and Fig. 3 the errors in Tables 3 and 4, obtained for different values of $\tau$.

We can observe that, with smaller values of $R$, our method performs better than the composite Simpson's rule, presented in [21], but for higher values of $R$ errors grow. 
Table 3: Errors in the case $\lambda=t_{[R / 4]}+h / 2+\tau h$, with $\tau=0,1 / 4$.

\begin{tabular}{lllll}
\hline $\mathrm{R}$ & $\tau=0$ & \multicolumn{3}{c}{$\tau=1 / 4$} \\
\hline & $\left|E_{R}^{S}\right|$ & $\left|E_{R}\right|$ & $\left|E_{R}^{S}\right|$ & $\left|E_{R}\right|$ \\
\hline 8 & - & $1.43 \mathrm{e}-05$ & - & $2.73 \mathrm{e}-02$ \\
16 & - & $1.20 \mathrm{e}-06$ & - & $6.83 \mathrm{e}-03$ \\
32 & - & $8.73 \mathrm{e}-08$ & - & $1.71 \mathrm{e}-03$ \\
64 & - & $3.66 \mathrm{e}-09$ & - & $4.27 \mathrm{e}-04$ \\
128 & - & $1.55 \mathrm{e}-06$ & - & $1.04 \mathrm{e}-04$ \\
256 & $6.40 \mathrm{e}-02$ & $2.79 \mathrm{e}-05$ & $3.16 \mathrm{e}-02$ & $9.47 \mathrm{e}-05$ \\
512 & $3.22 \mathrm{e}-02$ & $2.49 \mathrm{e}-04$ & $1.60 \mathrm{e}-02$ & $3.05 \mathrm{e}-03$ \\
1024 & $1.62 \mathrm{e}-02$ & $2.63 \mathrm{e}-02$ & $8.07 \mathrm{e}-03$ & $2.22 \mathrm{e}-02$ \\
2048 & $8.11 \mathrm{e}-03$ & $2.50 \mathrm{e}-01$ & $4.05 \mathrm{e}-03$ & $3.06 \mathrm{e}+00$
\end{tabular}

Table 4: Errors in the case $\lambda=t_{0}+h / 2+\tau h$, with $\tau=0,1 / 4$.

\begin{tabular}{lllll}
\hline $\mathrm{R}$ & $\tau=0$ & \multicolumn{3}{l}{$\tau=1 / 4$} \\
\hline & $\left|E_{R}^{S}\right|$ & $\left|E_{R}\right|$ & $\left|E_{R}^{S}\right|$ & $\left|E_{R}\right|$ \\
\hline 8 & - & $3.29 \mathrm{e}-04$ & - & $2.75 \mathrm{e}-02$ \\
16 & - & $8.26 \mathrm{e}-05$ & - & $6.88 \mathrm{e}-03$ \\
32 & - & $2.07 \mathrm{e}-05$ & - & $1.72 \mathrm{e}-03$ \\
64 & - & $5.53 \mathrm{e}-06$ & - & $3.93 \mathrm{e}-04$ \\
128 & - & $6.31 \mathrm{e}-05$ & - & $1.31 \mathrm{e}-03$ \\
256 & $1.26 \mathrm{e}-01$ & $1.31 \mathrm{e}-04$ & $6.32 \mathrm{e}-02$ & $3.91 \mathrm{e}-02$ \\
512 & $6.35 \mathrm{e}-02$ & $1.59 \mathrm{e}-01$ & $3.18 \mathrm{e}-02$ & $1.64 \mathrm{e}+00$ \\
1024 & $3.18 \mathrm{e}-02$ & $2.16 \mathrm{e}+00$ & $1.60 \mathrm{e}-02$ & $5.30 \mathrm{e}+01$ \\
2048 & $1.59 \mathrm{e}-02$ & $4.70 \mathrm{e}+00$ & $7.99 \mathrm{e}-03$ & $2.30 \mathrm{e}+02$ \\
\hline
\end{tabular}



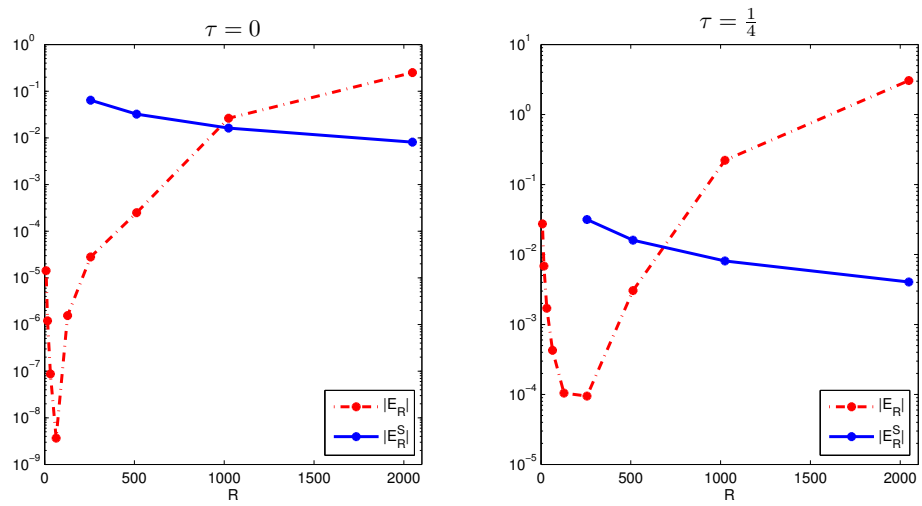

Figure 2: Graphical representation of absolute errors, in the case $\lambda=t_{[R / 4]}+h / 2+$ $\tau h$. Axis $y$ is in logarithmic scale.
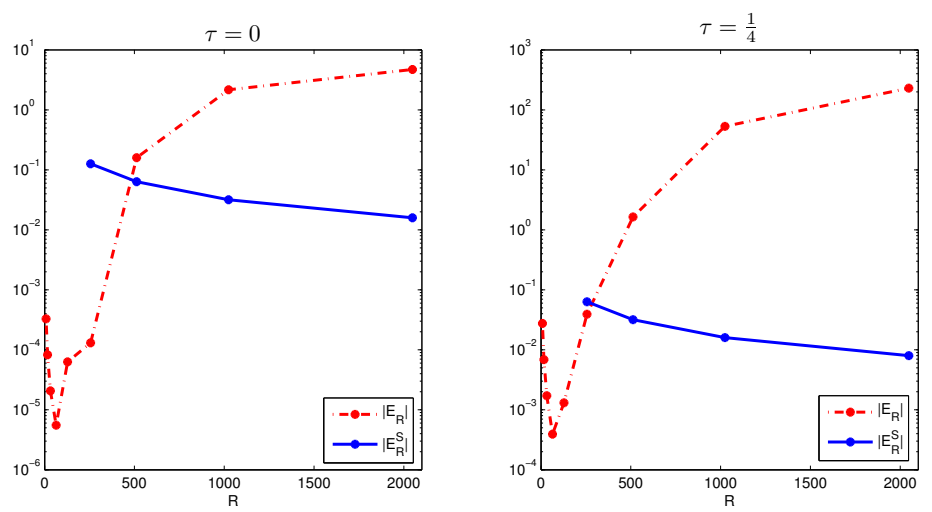

Figure 3: Graphical representation of absolute errors, in the case $\lambda=t_{0}+h / 2+\tau h$. Axis $y$ is in logarithmic scale. 
Table 5: Errors in the case $\delta=4$.

\begin{tabular}{lllll}
\hline $\mathrm{R}$ & $\gamma=1 / 3$ & \multicolumn{3}{l}{$\gamma=1 / 2$} \\
\hline & $\left|E_{R}^{N}\right|$ & $\left|E_{R}\right|$ & $\left|E_{R}^{N}\right|$ & $\left|E_{R}\right|$ \\
\hline 15 & $2.52 \mathrm{e}-02$ & $2.33 \mathrm{e}-04$ & $2.35 \mathrm{e}-02$ & $2.13 \mathrm{e}-04$ \\
33 & $2.17 \mathrm{e}-03$ & $1.89 \mathrm{e}-05$ & $1.98 \mathrm{e}-03$ & $1.65 \mathrm{e}-05$ \\
69 & $2.21 \mathrm{e}-04$ & $1.85 \mathrm{e}-06$ & $2.00 \mathrm{e}-04$ & $1.58 \mathrm{e}-06$ \\
141 & $2.44 \mathrm{e}-05$ & $1.97 \mathrm{e}-07$ & $2.20 \mathrm{e}-05$ & $1.68 \mathrm{e}-07$ \\
285 & $2.81 \mathrm{e}-06$ & $2.35 \mathrm{e}-08$ & $2.54 \mathrm{e}-06$ & $2.02 \mathrm{e}-08$
\end{tabular}

\subsection{Comparison with Newton-Cotes}

We evaluate numerically the finite-part integral (3) with $\alpha=\beta=0, p=1$, $\lambda=0$ and

$$
f(x)=x^{4}+|x|^{\delta+\gamma}, \quad \delta=3,4, \quad \gamma=\frac{1}{2}, \frac{1}{3} .
$$

Obviously, $f(x) \in C^{\delta+\gamma}([-1,1])$. In particular, we compare our quadrature (29) with Newton-Cotes rule of degree 3 [19]. The exact values of the integrals are [19]

$$
\begin{aligned}
& I(f ; 0 ; 1)=\frac{12+2 \gamma}{9+3 \gamma}, \text { for } \delta=4, \\
& I(f ; 0 ; 1)=\frac{10+2 \gamma}{6+3 \gamma}, \text { for } \delta=3,
\end{aligned}
$$

with $\gamma=1 / 2,1 / 3$. For both methods we adopt a uniform mesh.

To construct the piecewise Lagrange interpolation polynomial of degree $k$, we have to introduce $k-1$ quadrature nodes at each subinterval, then, in order to compare our method with Newton-Cotes, we have to assume $R=k n$, where $n$ is the number of subintervals used for the construction of the composite rule.

Let $E_{R}^{N}$ be the quadrature error obtained using the composite Newton-Cotes rule of degree 3, with $R+1$ quadrature nodes.

In Tables 5 and 6 we compare the results presented in [19] with the results obtained by our spline quadrature (29) for both values of $\delta$, then we represent the absolute errors in Fig. 4 and Fig. 5, so that we can compare graphically the behaviour of quadrature errors.

In both cases, we can see that our method perform better than the rule proposed in [19]. In particular, we observe that the accuracy of our spline quadrature with $R=69$ is comparable or sometimes better than the accuracy achieved by composite Newton-Cotes with $R=285$. 
Table 6: Errors in the case $\delta=3$.

\begin{tabular}{lllll}
\hline $\mathrm{R}$ & $\gamma=1 / 3$ & \multicolumn{3}{l}{$\gamma=1 / 2$} \\
\hline & $\left|E_{R}^{N}\right|$ & $\left|E_{R}\right|$ & $\left|E_{R}^{N}\right|$ & $\left|E_{R}\right|$ \\
\hline 15 & $5.11 \mathrm{e}-02$ & $9.60 \mathrm{e}-04$ & $4.43 \mathrm{e}-02$ & $3.60 \mathrm{e}-04$ \\
33 & $7.15 \mathrm{e}-03$ & $1.60 \mathrm{e}-04$ & $5.49 \mathrm{e}-03$ & $5.53 \mathrm{e}-05$ \\
69 & $1.18 \mathrm{e}-03$ & $2.93 \mathrm{e}-05$ & $7.99 \mathrm{e}-04$ & $9.25 \mathrm{e}-06$ \\
141 & $2.11 \mathrm{e}-04$ & $5.61 \mathrm{e}-06$ & $1.26 \mathrm{e}-04$ & $1.61 \mathrm{e}-06$ \\
285 & $3.96 \mathrm{e}-05$ & $1.09 \mathrm{e}-06$ & $2.07 \mathrm{e}-05$ & $2.82 \mathrm{e}-07$ \\
\hline
\end{tabular}
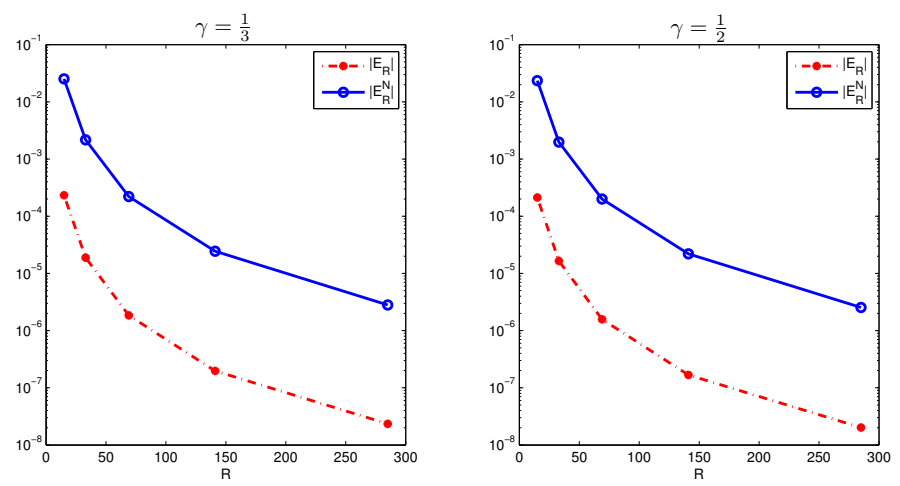

Figure 4: Graphical representation of absolute errors in the case $\delta=4$. Axis $y$ is in logarithmic scale.
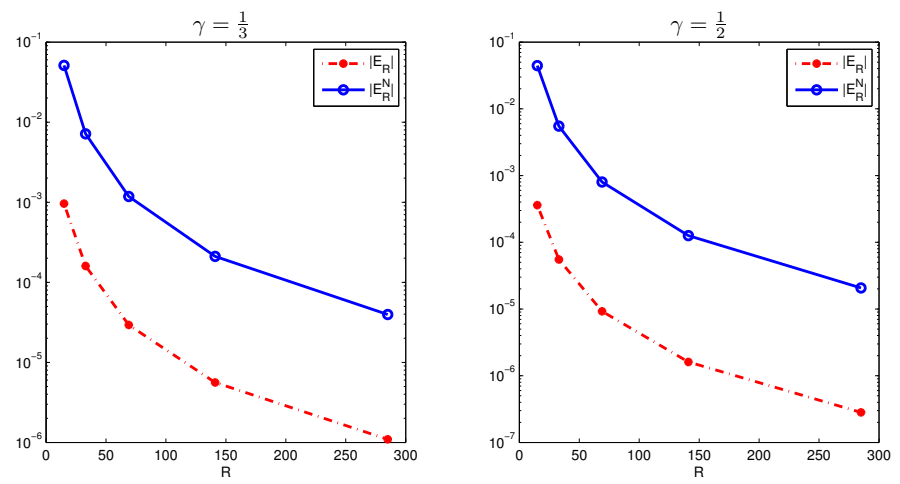

Figure 5: Graphical representation of absolute errors in the case $\delta=3$. Axis $y$ is in logarithmic scale. 


\subsection{Final remarks}

Evaluation of Hadamard finite-part integrals of the form (1) is reduced to the computation of Cauchy principal value integrals in virtue of Property 1 in Section 1 .

Property 2 in Section 1 says that when we subdivide the integration's interval $[a, b]$ in $R$ subintervals and when the singularity lies in one of these subintervals, the finite-part integral in Property 2 tends to infinity as $R \rightarrow \infty$. Moreover, the best case is when the singularity is located in the middle point of a subinterval [10].

From numerical results in Tables 2, 3 and 4 we can observe that our method presents the numerical instability phenomenon derived from Property 2 for values of $R$ smaller with respect to the considered composite rules. This behaviour is due to the fact that both quadratures (29) and (30) require integral evaluations on intervals $\left[t_{j}, t_{j+1}\right], j=0,1, \ldots, 3 R-1$. Consequently, for equally spaced knots and for fixed $R$, the integration interval containing the singularity is the third part of the integration interval required by other methods.

When the singularity is located in the middle point between two spline knots, as for example $\lambda=0$ in Section 5.1 and $\tau=0$ in Section 5.2, our quadrature performs slightly better for higher number of knots.

Nevertheless, by using a considerably less number of quadrature nodes, our spline quadrature achieves the same or better accuracy with respect to the considered composite rules. Moreover, we have a great flexibility in the choice of quadrature nodes and we can use spline spaces $S_{n+1}\left(T_{R}\right)$ of different orders.

The proposed quadrature cannot be extended to the case of finite-part integrals with endpoint singularities. Since Martensen interpolation is a natural extension of piecewise linear interpolation, the method should also be investigated for endpoint singularities as considered by C.W. Groetsch [7].

\section{References}

[1] De Villiers, J.M.: A convergence result in nodal spline interpolation. J. Approx. Theory 74, 266-279 (1993)

[2] De Villiers, J.M., Rowher, C.H.: Optimal local spline interpolants. J. Comput. Appl. Math. 18, 107-119 (1987)

[3] De Villiers, J.M., Rowher, C.H.: A nodal spline generalization of the Lagrange interpolant. In: Nevai, P., Pinkus, A. (eds.) Progress in Approximation Theory, pp. 201-211. Academic Press, Boston (1991) 
[4] Demichelis, V., Rabinowitz, P.: Finite-part integrals and modified splines, BIT 44, 259-267 (2004)

[5] Demichelis, V., Sciarra, M.: Smoothness of Martensen splines. Submitted

[6] Gerasoulis, A.: Piecewise-polynomial quadratures for Cauchy singular integrals. SIAM J. Numer. Anal. 23, 891-902 (1986)

[7] Groetsch, C.W.: Regularized Product Integration for Hadamard Finite Part Integrals. Computers Math. Appl. 30, 129-135 (1995)

[8] Lyche, T., Schumaker, L.L.: Local spline approximation methods. J. Approx. Theory 15, 294-325 (1975)

[9] Martensen, E.: Darstellung und Entwicklung des Restgliedes der Gregoryschen Quadraturformel mit Hilfe von Spline-Funktionen. Numer. Math. 21, 70-80 (1973)

[10] Monegato, G.: Numerical evaluation of hypersingular integrals. J. Comput. Appl. Math. 50, 9-31 (1994)

[11] Monegato, G.: Definitions, properties and applications of finite-part integrals. J. Comput. Appl. Math. 229, 425-439 (2009)

[12] Rabinowitz, P.: Uniform convergence results for Cauchy principal value integrals. Math. Comput. 56, 731-740 (1991)

[13] Rabinowitz, P.: Uniform Convergence Results for Finite-Part Integrals. Workshop on Analysis celebrating the 60th birthday of Pter Vrtesi and in memory of Ottó Kis and Árpad Elbert, Alfrd Rnyi Institute of Mathematics, Budapest (2001)

[14] Schumaker, L.L.: Spline functions: basic theory. Wiley, New York (1981)

[15] Siewer, R.: Martensen splines. BIT 46, 127-140 (2006)

[16] Siewer, R.: A constructive approach to nodal splines. J. Comput. Appl. Math. 203 , 289-308 (2007)

[17] Siewer, R.: Nodal splines on compact intervals. BIT 53, 741-753 (2013)

[18] Timan, A.F.: Theory of approximation of functions of a real variable. Dover Publications, Inc., New York (1994) 
[19] Wu, J., Sun, W.: The superconvergence of Newton-Cotes rules for the Hadamard finite-part integral on an interval. Numer. Math. 109, 143-165 (2008)

[20] Wu, J., Dai, Z., Zhang, X.: The superconvergence of the composite midpoint rule for the finite-part integral. J. Comput. Appl. Math. 233, 1954-1968 (2010)

[21] Zhang, X., Wu, J., Yu, D.: Superconvergence of the composite Simpson's rule for a certain finite-part integral and its applications. J. Comput. Appl. Math. 223, 598-613 (2009) 